\title{
Verläufe von Traumafolgen bei ehemaligen politisch Inhaftierten der DDR
}

\author{
Maercker, Andreas ; Gäbler, Ira ; Schützwohl, Matthias
}

\begin{abstract}
Hintergrund In einer Mitte der 1990er Jahre untersuchten Stichprobe ehemaliger politisch Inhaftierter der DDR wurden im 15-Jahres-Follow-up die Veränderungen der Diagnose- und Symptomprävalenzen der posttraumatischen Belastungsstörung (PTBS) sowie anderer psychischer Störungen untersucht. Zudem wurden die durch Kliniker erhobenen Diagnosenverläufe mit den retrospektiven subjektiven Einschätzungen der Studienteilnehmer verglichen. Methode Dreiundneunzig ehemals politisch inhaftierte Personen nahmen an der Folgestudie teil (85\% Wiederteilnahme), ihr mittleres Alter betrug 64 Jahre. Diagnosen und Symptome wurden mittels strukturierter klinischer Interviews sowie Fragebögen erfasst. Die retrospektiven subjektiven Verlaufseinschätzungen der Teilnehmer wurden mittels eines PTBS-Symptomindex basierend auf 4 Symptomgruppen (Intrusionen, Vermeidung, Numbing, Hyperarousal) errechnet. Ergebnisse Eine aktuelle PTBS lag bei 33\% vor (1997: 29\%). Nur ca. in der Hälfte der Fälle lag diese schon 1994 vor, bei der anderen Hälfte handelt es sich um neu-inzidente bzw. remittierte Fälle. Nächsthäufige Diagnosen waren Major-Depression, Episode (26\%), Panik (mit oder ohne Agoraphobie: 24\%) sowie somatoforme Störungen (19\%). Im PTBS-Symptomprofil nahmen Intrusionen, Flashbacks bzw. Entfremdungsgefühl im Zeitverlauf ab, Reizbarkeit und Schreckreaktionen hingegen zu. Die subjektive Selbsteinschätzung der PTBS-Symptomverläufe durch die Studienteilnehmer ergab im Vergleich mit den Diagnostikern häufiger ein resilientes („nie PTBS“) oder verzögertes und seltener ein remittiertes Verlaufsmuster. Schlussfolgerung Die Ergebnisse sprechen für eine traumabezogene Langzeitmorbidität, die allerdings instabiler ist als bisher angenommen. BACKGROUND: In a sample of ex-political prisoners in the GDR examined in the middle of the 1990s, a follow-up examination carried out 15 years later investigated alterations in the diagnosis and symptom prevalence of post-traumatic stress syndrome PTSS) as well as other psychiatric disorders. The diagnosis course given by the clinicians was compared with the retrospective subjective estimations by participants. METHODS: A total of 93 ex-political prisoners participated in the follow-up study (85\%) with an average age of 64 years. Diagnoses and symptoms were collated using structured clinical interviews and questionnaires. The retrospective subjective estimation of progression of the participants was calculated using a PTSS symptom index based on four symptom groups (intrusions, avoidance, numbing and hyperarousal). RESULTS: At the time of follow-up PTSS was present in $33 \%$ of participants (in 1997 it was $29 \%$ ). In 1994 this was present in only approximately $50 \%$ of cases and in the other $50 \%$ it concerned newly incident or remission cases. The next most common diagnoses were major depression episodes (26\%), panic (with or without agoraphobia $24 \%$ ) and somatoform disorders (19\%). In the PTSS symptom profile intrusions, flashbacks and alienation feelings decreased with time but in contrast irritability and shock reactions increased. The subjective self-estimation of the course of PTSS symptoms by the participants often gave a resilient (never PTSS) or delayed and rarely a remission course pattern in comparison to the diagnosing clinicians. CONCLUSIONS: The results indicate a trauma-related long-term morbidity which is, however less stable than previously assumed.
\end{abstract}

DOI: https://doi.org/10.1007/s00115-012-3646-y

Other titles: Course of trauma sequelae in ex-political prisoners in the GDR : A 15-year follow-up study 
Posted at the Zurich Open Repository and Archive, University of Zurich

ZORA URL: https://doi.org/10.5167/uzh-66236

Journal Article

Accepted Version

Originally published at:

Maercker, Andreas; Gäbler, Ira; Schützwohl, Matthias (2013). Verläufe von Traumafolgen bei ehemaligen politisch Inhaftierten der DDR. Der Nervenarzt, 84(1):72-78.

DOI: https://doi.org/10.1007/s00115-012-3646-y 
Verläufe von Traumafolgen bei ehemaligen politischen Inhaftierten der DDR. Ein 15Jahres-Follow-up

Andreas Maercker, Ira Gäbler \& Matthias Schützwohl

Version: 03.07.2012 


\section{Abstract}

Hintergrund. In einer Mitte der 1990er Jahre untersuchten Stichprobe ehemaliger politischer Inhaftierter der DDR wurden im 15-Jahres-Follow-up die Veränderungen der Diagnose- und Symptom-Prävalenzen der Posttraumatischen Belastungsstörung (PTBS) sowie anderer psychischer Störungen untersucht. Zudem wurden die durch Kliniker erhobenen Diagnosenverläufe mit den retrospektiven subjektiven Einschätzungen der Studienteilnehmer verglichen.

Methode. 93 ehemals politisch inhaftierte Personen nahmen an der Folgestudie teil (85\% Wiederteilnahme), ihr mittleres Alter betrug 64 Jahre. Diagnosen und Symptome wurden mittels strukturierter klinischer Interviews sowie Fragebögen erfasst. Die retrospektiven subjektiven Verlaufseinschätzungen der Teilnehmer wurden mittels eines PTBSSymptomindex basierend auf vier Symptomgruppen (Intrusionen, Vermeidung, Numbing, Hyperarousal) errechnet.

Ergebnisse. Eine aktuelle PTBS lag bei 33\% vor (1997: 29\%). Nur ca. in der Hälfte der Fälle lag diese schon 1994 vor, bei der anderen Hälfte handelt es sich um neu-inzidente bzw. remittierte Fälle. Nächsthäufige Diagnosen waren Major Depression, Episode (26\%), Panik (mit oder ohne Agoraphobie: 24\%) sowie somatoforme Störungen (19\%). Im PTBSSymptomprofil nahmen Intrusionen, Flashbacks bzw. Entfremdungsgefühl im Zeitverlauf ab, Reizbarkeit und Schreckreaktionen hingegen zu. Die subjektive Selbsteinschätzung der PTBS-Symptomverläufe durch die Studienteilnehmer ergab im Vergleich mit den Diagnostikern häufiger ein resilientes (,nie PTBS“) oder verzögertes und seltener ein remittiertes Verlaufsmuster.

Schlussfolgerung. Die Ergebnisse sprechen für eine trauma-bezogene Langzeitmorbidität, die allerdings instabiler ist, als bisher angenommen. 
Politische Verfolgung und insbesondere politisch bedingte Inhaftierungen gelten unbestritten als potenziell traumatische Ereignisse, die zur Posttraumatischen Belastungsstörung (engl.: PTSD) und zu anderen Traumafolgestörungen führen können. Eine Reihe von Studien belegt ein hohes Ausmaß psychischer Beeinträchtigungen bei ehemaligen politischen Gefangenen der DDR (1949 - 1989) (z.B. Priebe et al., 1993; Maercker \& Schützwohl, 1997; Weissflog et al., 2011). In diesen Studien mit insgesamt ca. 500 Personen fanden sich PTBS-Prävalenzen von 22-30\%. In der aktuellsten dieser Studien (Weissflog et al., 2011) wurde eine PTBS-Prävalenz von 50\% berichtet. Die Studie konnte allerdings nicht klären, ob diese vergleichsweise hohe PTBS-Prävalenz eine generelle Erhöhung haftbedingter psychischer Störungen im zeitlichen Abstand zum Ende der DDR oder durch methodische Besonderheiten der Studie (Selbsteinschätzung der Symptome und Querschnittsdesign) mitbedingt ist. International ist das Phänomen scheinbar ansteigender PTBS-Prävalenzen mit steigendem Lebensalter bei Überlebenden von traumatischen Ereignissen bereits mehrfach beschrieben. Solomon (1994) und Prot (2009) konnten einen Anstieg verschiedener traumabedingter Psychopathologien bei Israelischen Überlebenden des Holocausts mehrere Jahrzehnte nach dessen Ende zeigen. Dieser Anstieg ist umso bemerkenswerter, da parallel zur Einführung der PTBS-Diagnose in die Störungsklassifikationsverzeichnisse DSM-III und ICD-10 das therapeutische Wissen sprunghaft wuchs und heute eine größere Anzahl wirksamer Therapien vorliegt (Flatten et al., 2011). Auch vielen der ehemaligen politischen Inhaftierten der DDR wurden in den letzten 20 Jahren Psychotherapien angeboten (Freyberger, Frommer, Maercker \& Steil, 2003).

Frühere politisch Inhaftierte aus der DDR sind zudem eine Gruppe vergleichsweise älterer Menschen, in der Regel sind sie über 60 Jahre alt. In den meisten internationalen Studien zur PTBS werden dagegen jüngere Personen untersucht. Bisher ist für PTBS noch 
weitgehend ungeklärt, wie diese sich typischerweise im höheren Lebensalter entwickelt (Lapp et al., 2011). Es gibt einige Befunde und Annahmen, die - ähnlich wie bei der Major Depression - bei PTBS von einer Altersabnahme ausgehen (Kessler et al., 2005). Andere Studien und biopsychosoziale Konzepte legen nahe, dass es im Alter nicht zu (spontaner) Abnahme der PTBS-Symptomatik kommt, sondern eher zu einem Umbau im Symptomprofil mit z.B. trauma-bezogenen Schlafstörungen und erhöhter Wachsamkeit (Lapp et al., 2011, Maercker, 1999).

In der Mitte der 1990er Jahre hatte eine Finanzierung des Bundesministeriums für Bildung und Forschung es ermöglicht, in Dresden eine Untersuchung ehemaliger politischer Inhaftierter der DDR zwischen 1949 und 1989 durchzuführen (z.B. Maercker \& Schützwohl, 1997). Dabei wurden anhand strukturierter klinischer Interviews und von Fragebögen eine Gruppe von 146 ehemaligen Inhaftierten über Kontakte mit Opfervereinen und der Presse untersucht und diese mit einer soziodemografisch parallelisierten Kontrollgruppe verglichen. Für alle Untersuchten lagen traumatische Erlebnisse vor (Folter, Gewalterlebnisse oder androhungen, Einzelhaft u.a.; vgl. Maercker, Beauducel \& Schützwohl, 2000). Neben der PTBS als häufigste Traumafolge-Erkrankung zeigte sich, dass soziale Phobien, Substanzmissbrauch und spezifische Phobien (die meisten in Form einer Klaustrophobie) signifikant häufiger bei den Ex-Häftlingen auftraten als bei der Kontrollgruppe. Die Depressions- und allgemeinen Psychopathologiewerte beider Gruppen unterschieden sich signifikant, mit einem BDI-Gruppenmittelwert bei den Ex-Häftlingen von 14 Punkten, der damit im Bereich milder Depressionen lag. Das mittlere Alter der Untersuchten war damals 54 Jahre.

Im Jahr 2008 wurde es möglich, eine Folgeuntersuchung des Samples der ehemaligen politischen Inhaftierten durchzuführen (ohne wiederholte Untersuchung der Kontrollgruppe). Hauptfragestellungen dieser Erhebung waren: 
- Haben sich die Prävalenzen klinischer Störungen in diesem 15-Jahreszeitraum verändert?

- Wie hat sich das Profil der PTBS-Symptome über die Zeit verändert?

- Stimmen die retrospektiven Angaben der Studienteilnehmer über ihre Störungsverläufe (subjektives Bild) mit den 1994 und 2008 von Klinikern beurteilten Störungsverläufen (klinisches Bild) überein?

\section{Methode}

\section{Studienpopulation und Design}

In der Mitte der 1990er Jahre wurde eine Stichprobe nicht-behandlungssuchender ehemaliger politischer Inhaftierter der DDR untersucht, die durchschnittlich 29.3 Monate (SD: 28.5) in Haft gewesen waren (Maercker \& Schützwohl, 1997). Die längsschnittliche Fortführung war den Teilnehmern in den 1990er Jahren nicht angekündigt, daher mussten die in einem zeithistorischen Institut bereits archivierten Studienteilnehmerdaten für die Folgeuntersuchung herausgesucht werden. Nach einem schwierigen Prozess der AdressBestätigung bzw. -Neusuche wurde der Personenkreis kontaktiert und zur neuen Untersuchung eingeladen (ca. 40\% der Teilnehmer hatten in der Zwischenzeit neue Adressen). Die Folgeinterviews fanden 2008 entweder in der Universitätsklinik für Psychiatrie und Psychotherapie in Dresden statt oder in einigen Fällen aufgrund von Mobilitätsproblemen in den Heimatorten der Teilnehmer.

Von den 146 ursprünglichen Teilnehmern konnten 11 nicht lokalisiert werden und 25 der Studienteilnehmer, die lokalisiert werden konnten, waren bereits verstorben. Von den verbliebenen 110 ehemaligen Studienteilnehmern waren 17 (15.5\%) nicht zur Teilnahme bereit (hauptsächlich aufgrund von Gesundheitsproblemen). Daher umfasst die vorliegende Studie 93 Teilnehmer (d.h. es bestand eine Ausschöpfungsrate von 84.5\% der noch lebenden lokalisierten ursprünglichen Teilnehmer). Sieben Personen nahmen nur am Fragebogenteil der 
Studie teil. Zum Zeitpunkt der vorliegenden Studie (2008) waren die Teilnehmer 40 bis 85

Jahre alt $(M=64.4$ Jahre; SD = 10.7). Die mittlere Zeit seit der Haftentlassung war 36.8 Jahre $(\mathrm{SD}=10.9)$

Die wichtigsten Stichprobenmerkmale der Teilnehmer an beiden Untersuchungsszeitpunkten findet sich in Tabelle 1 (erste Spalte). Um die Drop-out-Faktoren abzuschätzen, wurden alle Variablen in logistische Regressionsmodelle aufgenommen. Erwartungsgemäß war höheres Alter mit den Drop-outs assoziiert $(\mathrm{OR}=2.04,95 \%, \mathrm{KI}=1.15$ 3.62), zudem geringere Bildung $(\mathrm{OR}=.36,95 \%, \mathrm{KI}=.14-.89)$. Alle anderen Variablen waren keine Prädiktoren.

---Tabelle 1 bitte hier einfügen---

Die verwendeten Daten entsprechen einem retro- und prospektiven Längsschnitts-Design: Zusätzlich zu den prospektiven Daten von 1994-1995 (T1) und 2008 (T2) wurde ein T0 als der Zeitraum direkt nach der individuellen Haftentlassung spezifiziert, und 1994-1995 bereits mit erfragt.

\section{Psychometrische Erhebung}

PTBS und Diagnosen weiterer Störungskategorien wurden zu T1 und T2 durch erfahrene klinische Beurteiler mittels des "Diagnostischen Interview für psychische Störungen: DIPS” (Schneider \& Margraf, 2006) untersucht und beurteilt. Das DIPS ist ein an den jeweiligen DSM-Versionen ausgerichtetes Erhebungsinstrument, das alle affektiven, Angst- und somatoformen Störungen erfasst und die Substanzabhängigkeiten screent; es hat hervorragende Reliabilitäts- und Validitätskennwerte (Schneider \& Margraf, 2006).

Die 17 PTBS-Symptome laut DSM-IV wurden jeweils im PTBS-Teil des DIPS erhoben. Die retrospektive T0-Diagnose wurde zu T1 mit folgender Instruktion erfasst: "War dieses 
[Symptom] im ersten Jahr nach Ihrer Haftentlassung bei Ihnen vorhanden? Wenn ja, wie lange?”. PTBS-Diagnosen wurden nach dem DSM-III-R bzw. IV-Algorithmus anhand der als vorhanden ermittelten Symptome vergeben.

Längsschnittliche Diagnoseverläufe: Da Daten aus drei Zeitpunkten Verwendung finden, ergeben sich vor dem Hintergrund vorliegender binärer Ausprägungen (PTBS nicht vorhanden vs. PTBS vorhanden) acht mögliche Muster, die sich klinisch auf fünf Verlaufstypen reduzieren lassen: chronisch (stets PTBS vorhanden); resilient (PTBS stets nicht vorhanden); verzögert, zusammengesetzt aus früh verzögert (T0: nicht; T1: vorhanden; T2: vorhanden) sowie spät verzögert (T0: nicht; T1: nicht; T2: vorhanden); remittiert zusammengesetzt aus früh remittiert (T0: vorhanden; T1: nicht; $\mathrm{T} 2$ : nicht) und spät remittiert (T0: vorhanden; T1: vorhanden; T2: nicht); und wechselnd zusammengesetzt aus einem Uförmigen Verlauf (T0: nicht; T1: vorhanden; T2: nicht) und einem invers U-förmigen Verlauf (T0: vorhanden; T1: nicht; T2: vorhanden).

Die Kliniker-Beurteilungen konnten direkt für diese Einteilung herangezogen werden. Die subjektiven retrospektiven Einschätzungen der Teilnehmer wurden durch eine graphische Methode erhoben. Hierbei waren vier Diagramme vorgegeben, eines pro PTBSSymptomgruppe: Intrusionen, Vermeidung, Numbing, Hyperarousal). Die 4Symptomgruppen-Einteilung wurde gewählt, da die PTBS-Grundlagenforschung überwiegend von einer 4-Faktor-Lösung dieser Symptomatik ausgeht (Elhai \& Palmieri, 2011; vgl. dagegen Morina et al., 2010). Für jede Symptomgruppe wurde ein deutscher Begriff gewählt, der auf den Arbeitsblättern kurz erläutert wurde (Wiedererleben der/des Ereignisses, bewusstes Vermeiden, emotionale Abgestumpftheit, Angespanntheit). Auf der Abszisse waren 3 Zeitanker vorgegeben (,direkt nach der Haftentlassung“, „1990er Jahre“ und „heute“). Die Ordinate war eine 8-stufige visuelle Analogskala, die mit „Schweregrad“ 
bezeichnet war. Die Probanden hatten die Instruktion, freihändig den ungefähren Verlauf ihrer Symptomatik einzuzeichnen.

Die zu den 3 spezifizierten Zeitpunkten eingezeichneten Schätzwerte wurden pro Teilnehmer und Zeitpunkt gemittelt und dann am Median binär geteilt (,geringe Belastung“: 0-3,49 ; „hohe Belastung“: 3,5-7). Die Zuordnung zu den acht Diagnoseverläufen wurde anhand der Binärwerte (geringe vs. hohe Belastung) vorgenommen, die dem ,nicht vorhanden“ vs. „,vorhanden“ bei der PTBS-Diagnosestellung der Kliniker entspricht.

Therapie-Inanspruchnahme: Es wurde erfragt, ob "aufgrund der erfragten Erlebnisse und psychischen Beschwerden eine spezifische ärztliche oder psychologische Hilfe in Anspruch genommen" worden war oder wird. Da nur sechs der 86 Befragten (7\%) diese Frage bejahten, wurde diese Variable aufgrund dieser niedrigen Basisrate nicht in die folgenden Auswertungen einbezogen.

\section{Datenanalysen}

Die Prävalenzraten und Symptomhäufigkeiten wurden mittels $\mathrm{Chi}^{2}$-Statistik getestet. Die Beurteiler-Übereinstimmungen im ersten Schritt ebenfalls mittels $\mathrm{Chi}^{2}$-Statistik und nachfolgend anhand Treffer-Kombinatorik mittels Sensitivitäts- und Spezifitätswerten und deren 95\%-Konfidenzintervallen (95\%-KI) untersucht.

\section{Ergebnisse}

\section{Störungs-Prävalenzen}

Zum Zeitpunkt der Folgeuntersuchung 2008 hatten 32.6\% der Befragten eine Posttraumatische Belastungsstörung. Die Prävalenzrate unterscheidet sich damit nicht signifikant von der, die 1994 für diese Stichprobe ermittelt worden war (29.1\%) (Tab. 2). Zusätzlich fand sich bei $25.6 \%$ eine subsyndromale PTBS (vgl. Schützwohl \& Maercker, 1999); 1994 waren es $24.4 \%$ mit partieller PTBS. Die retrospektive Diagnostik für T0 (kurz 
nach Haftentlassung) hatte 1994 Prävalenzraten von 61.6\% für PTBS und 20.9\% für subsyndromale PTBS ergeben.

Als Störung mit der zweithöchsten Prävalenz erwies sich die Major Depression, einzelnen Episode mit 25.6\%, welche sich signifikant von der Prävalenzrate für Depressionen zu T1 unterscheidet (1994: 5.8\%). Signifikant geringer geworden sind die Prävalenzen von Klaustrophobien (als Form der spezifischen Phobien) und des substanzbezogenen Störungen.

---Tabelle 2 bitte hier---

\section{PTBS-Symptomprofile}

Bei der klinischen Symptomerhebung durch den Diagnostiker (s. Abb. 1) nahm die Prävalenz von zwei Intrusionssymptomen ab (Wiederkehrende Bilder, McNemar $\chi^{2}=9.53$, $\mathrm{df}=1, \mathrm{p}<0.01$; Flashbacks, McNemar $\left.\chi^{2}=6.55, \mathrm{df}=1, \mathrm{p}<0.05\right)$ während sich die von drei Intrusionssymptomen statistisch nicht signifikant verringerte (Belastende Träume, Belastung bei Erinnerung, Körperliche Reaktionen bei Erinnerung). Von den Vermeidungs/Betäubungs-Symptomen trat in der Nacherhebung nur das Entfremdungsgefühl signifikant seltener auf $\left(\right.$ McNemar $\left.\chi^{2}=6.76, \mathrm{df}=1, \mathrm{p}<0.05\right)$, während die anderen sechs Symptome statistisch gleich häufig blieben. Von den Hyperarousalsymptomen erhöhten sich die Prävalenzraten der Reizbarkeit $\left(\right.$ McNemar $\left.\chi^{2}=4.57, \mathrm{df}=1, \mathrm{p}<0.05\right)$ und der Schreckreaktion $\left(\operatorname{McNemar} \chi^{2}=5.56, \mathrm{df}=1, \mathrm{p}<0.05\right)$.

--Abb. 1 bitte hier--

\section{Übereinstimmung der Diagnoseverläufe und subjektiven Verläufe}

Für die durch Kliniker festgestellten Verläufe erwiesen sich über die drei retro- und prospektiven Zeiträume das resiliente Verlaufsmuster (30.2\%) sowie das früh remittierte Verlaufsmuster (25.6\%) als die häufigsten (Tab. 3). 
---Tabelle 3 bitte hier einfügen---

In der mittels graphischer Erfassung ermittelten subjektiven Verlaufseinschätzung hatten bezogen auf T2 N=31 Studienteilnehmer (36.0\%) eine so hohe Störungsbelastung angegeben, dass dies einer PTBS-Diagnose entspricht; diese Teilnehmer teilten sich auf den chronischen, die verzögerten und den U-förmig wechselnden Verlauf auf. Ein resilienter Verlauf fand sich wie bei der Klinikerbeurteilung am häufigsten (36.0\%; vgl. Tab. 3).

Die Prüfung der Übereinstimmung zwischen Kliniker-Beurteilung und TeilnehmerSelbsteinschätzung wurde in mehreren Schritten vorgenommen. Zunächst wurden mittels Kreuztabelle der Anteil an den Kliniker-Diagnosen berechnet, der sich auch in der ProbandenSelbstbeurteilung fand (Tab. 3 letzte Spalte). Beim resilienten Verlaufstyp war demnach die Übereinstimmung mit $61.5 \%$ am höchsten, gefolgt vom chronischen Verlaufsmuster (42.9\%) und den beiden remittierten Verlaufsmustern (20.0-22.7\%). Die anderen Verlaufsmuster (verzögerte, wechselnde) zeigten weitgehend oder vollständig fehlende Übereinstimmung. Abschließend wurden die Wahrscheinlichkeiten berechnet, dass ein in der KlinikerBeurteilung PTBS-positiv eingeschätzter Teilnehmer sich auch in der Selbsteinschätzung PTBS-positiv sah (entspricht einer Sensitivitäts-Analyse). Für T1 lag die „Richtig-PositivRate“ bei 0.80 (95\%-KI: 0.61-0.91), d.h. 20 von 25 Probanden sahen sich selbst als PTBSpositiv, während für T2 diese Rate mit 0.64 (95\%-KI: 0.46-0.79) bei 18 von 28 Probanden niedriger ausfiel. Da die beiden 95\%-Konfidenzintervalle sich überlappen, unterscheiden sich die Werte nicht signifikant.

Die komplementäre Wahrscheinlichkeit, dass beide_-Kliniker und Selbstbeurteilende_mit ihrer Einschätzung des PTBS-negativ Status übereinstimmten (entspricht der Spezifitätsoder Richtig-Negativ-Rate), betrug zu T1 0.66 (40 von 61 Probanden) und war zu T2 mit 0.77 
(45 von 58 Probanden) höher. Das 95\%-KI zu T1 betrug 0.53-0.76 und zu T2 0.65-0.86, was wieder eine Überlappung und damit ein nicht signifikantes Ergebnis belegt.

\section{Diskussion}

Die vorliegende Studie hatte drei Ziele, (1) die Untersuchung der Diagnosestabilitäten über den 15-Jahres-Zeitraum, (2) die Untersuchung von Veränderungen im Symptomprofil der PTBS sowie (3) die Bewertung der Validität subjektiver retrospektiver PTBSVerlaufseinschätzungen.

Über die untersuchten ehemaligen politischen Inhaftierten der DDR waren bereits verschiedene klinische und wissenschaftliche Aspekte berichtet worden (Ehlers, Maercker \& Boos, 2000, Maercker, Bedauducel \& Schützwohl, 2000, Maercker \& Schützwohl, 1997, Schützwohl \& Maercker, 1999). Die vorliegende Folgestudie untersuchte zusätzlich weitere Aspekte als die hier berichteten (z.B. autobiografische Gedächtnisfunktionen: Kleim et al., 2012; Rachegedanken: Gäbler \& Maercker, 2011; Prädiktoren unterschiedlicher Verlaufstypen: Maercker et al., 2012; sozial-interpersonelle Faktoren: Krammer et al., 2012).

Die Untersuchung der Diagnosestabilitäten zeigt, insbesondere für die Posttraumatische Belastungsstörung, ein mehrschichtiges Bild. Auf den ersten Blick hat sich die PTBSPrävalenz über den 15-Jahreszeitraum nicht verändert (jeweils um 30\%). Bei genauerer Betrachtung zeigt sich allerdings, dass seit den 1990er Jahren ca. ein Drittel der Personen, die damals mit PTBS diagnostiziert wurden, ausgeheilt sind und dass etwa ein weiteres Drittel neu-/wieder-inzidente PTBS-Fälle sind, wie in Abbildung 1 grafisch veranschaulicht. Das Verlaufsmuster einer verzögerten PTBS, die erst im Zeitraum nach der ersten Untersuchung (T1) neu aufgetreten war, fand sich z.B. bei sechs Prozent der Studienteilnehmer, was in dieser Größenordnung der verzögerten PTBS-Entstehung bei anderen Traumatisiertengruppen entspricht (Brewin et al., 2007; Horesh et al., 2011). Zusätzliche Analysen zeigten, dass die verzögerte PTBS nicht mit potenziellen späteren traumatischen Ereignissen, der historischen 
Phase der Inhaftierung oder anderen anamnestischen Faktoren zusammenhängt sondern einzig mit einem höheren Bildungsstatus der Teilnehmer (Maercker et al., 2012). Insgesamt verbirgt sich also hinter der im Mittel gleichhohen PTBS-Prävalenz eine auch von anderen Störungsbildern bekannte Verlaufsdifferenzierung, die bereits in anderen Studien zum Langzeitverlauf der PTBS gezeigt werden konnte (z.B. Yehuda et al, 2009).

Bei den komorbiden Diagnosen ergaben sich veränderte Prävalenzraten mit einem Anstieg für die Major Depression (aktuelle Episode). Geringere Prävalenzraten nach 15 Jahren ergaben sich für die klaustrophobische Form der spezifischen Phobie (die 1994 mit $21 \%$ vergleichsweise hoch war) sowie den Substanzmissbrauch und -abhängigkeit. Die Verringerung der Klaustrophobiehäufigkeit ist unseres Wissens bisher noch nicht in anderen klinischen Stichproben beschrieben worden und lässt sich mit der prinzipiell guten Therapiebarkeit von spezifischen Phobien in Verbindung bringen (Ost et al., 2001); alternativ könnten sich zwischen den beiden Zeiträumen die Diagnosestellung etwas verändert haben. Der Befund geringer werdender Substanzmissbrauchs-Prävalenzen im Verlauf ist schwerer einzuordnen und hängt möglicherweise damit zusammen, dass Traumatisierte einen dysfunktionalen Alkoholkonsum im Sinne einer Selbstmedikation auftretender psychischer Beschwerden eher unmittelbar nach ihrer Traumatisierung zeigen (Schäfer, 2011).

Der Vergleich der PTBS-Symptomprofile über den 15-Jahreszeitraum ist zusätzlich zur allgemeinen Verlaufsbetrachtung auch vor dem Hintergrund zu interpretieren, dass die Probanden aktuell im Durchschnitt 64 Jahre alt waren. Eine neuere Übersicht legt theoretische Gründe für die Annahme nahe, dass sich das PTBS-Symptomprofil bei älteren Menschen verändert, z.B. kortikaler Disinhibition (Glück \& Maercker, 2012), ähnlich wie dies bei anderen psychischen Störungen der Fall ist. Direkte Belege für eine altersbezogene Zunahme PTBS-bezogener Schlafstörungen ergaben sich nicht, da diese nicht signifikant anstiegen. Auch die Frequenz belastender Träume oder Alpträume veränderte sich nicht signifikant. Insgesamt gab es allerdings einen Anstieg für 2 der 5 Hyperarousal-Symptome (Reizbarkeit 
und Schreckreaktion), was möglicherweise auf spezifische altersbezogene Veränderungen der Reagibilität hinweist (Uchino et al., 2010). Drei der übrigen Symptome verringerten sich signifikant: Wiederkehrende belastende Erinnerungen, Nachhallerinnerungen (Flashbacks) sowie das Entfremdungsgefühl. Die sehr wenigen anderen Studien zum Langzeit- bzw. Altersverlauf von PTBS-Symptomprofilen ergaben im Vergleich kaum signifikante und dazu uneinheitliche Befunde (Dirkzwanger et al., 2001, Solomon \& Mikulincer, 2006). Die vorliegende Studie belegt erstmals eindeutiger einen Symptomprofilwandel hin zu mehr Hyperarousal- und weniger Intrusions- bzw. Vermeidungssymptomen.

Die dritte Fragestellung dieser Untersuchung betraf die Validität subjektiver retrospektiver PTBS-Verlaufseinschätzungen der Probanden im Vergleich zu klinischdiagnostischen Beurteilungen aus den Mitte-1990er-Jahren und 15 Jahre später. Die Studienteilnehmer hatten vier verschiedene Verlaufsdiagramme für die Symptomgruppen Intrusionen, Vermeidung, Betäubung (Numbing) und Übererregung (Hyperarousal) gezeichnet, die zu einer gemeinsamen Symptombelastungslinie verrechnet wurden. Die Kliniker bzw. Diagnostiker hatten im Jahr 1994 retrospektive Symptombeurteilungen vorgenommen und seitdem zwei prospektive Einschätzungen.

Aus den Berechnungen zur Übereinstimmung der Kliniker-Beurteilungen (prospektiv erhoben zu T1 und T2) und der Probanden-Selbstbeurteilungen (zu T2 erhoben, d.h. retrospektiv für T1) ergab sich insgesamt, dass für die häufigen Verlaufsmuster tendenziell höhere Übereinstimmungen bestanden als für die selteneren, und dass die Probanden ihren Zustand retrospektiv in Richtung höherer PTBS-Belastung aggravierten. Im Detail hatte sich ergeben: Für den chronischen und resilienten (krankheitsfreien) Verlauf ergeben sich insgesamt ähnlich hohe Einschätzungen der Bestandsraten mit allerdings nur mittelhohen Übereinstimmungen für den individuellen Fall. D.h. sowohl die Einschätzung durch die Kliniker als auch die Selbsteinschätzung der Betroffene ergeben einen Anteil von ca. einem Drittel der Population, der als resilienter Typus angesehen werden kann. Dieses Urteil stimmt 
individuenbezogen allerdings nur bei $62 \%$ überein — die anderen $38 \%$ werden entweder nur von den Klinikern oder nur in der Selbstbeurteilung als resilient, d.h. „,immer gesund geblieben“, eingeschätzt. Die Kliniker schätzen dagegen insgesamt mehr Studienteilnehmer als „remittiert“ (gesundet) ein; die individuenbezogene Übereinstimmung liegt hier nur bei rund 20\%. Einen gewissen Trend zu einer retrospektiven Aggravationstendenz für den T1Zeitraum drückt sich in der abnehmenden Richtig-Positiv-Rate der Beurteilungsübereinstimmung (,Sensitivität“) und der steigenden Richtig-Negativ-Rate (,Spezifität“) aus (der allerdings nicht signifikant wird, wenn man den sehr konservativen Vergleich der Konfidenzintervalle zugrunde legt). Wieso es zu dieser Tendenz einer retrospektiv höheren und aktuell geringeren Aggravation kommt, muss offen bleiben. Diese können jedenfalls nicht im Sinne einer Intention zu mehr Entschädigungsleistungen zu gelangen, gesehen werden, denn sie betreffen ja einen früheren Zeitpunkt. Möglicherweise spielen psychologische Faktoren einer (negativen) Lebensbilanzierung hier eine Rolle (Maercker \& Horn, 2012).

Die vorliegende Studie hatte eine wichtige Einschränkung, da ihr Forschungsdesign retround prospektive Anteile kombinierte, wobei nur die für die prospektiven Zeitpunkte Erinnerungsverzerrungen ausgeschlossen werden können. Alle für T0 erhobenen Angaben unterliegen möglicherweise solchen Erinnerungsverzerrungen. Da Opfer politischer Gewalt allerdings fast nie direkt nach dem jeweiligen individuellen Gewaltende untersucht werden können (da sie dann noch in den gewaltanwendenden Ländern leben), ist hier keine andere Erhebungsmethodik möglich. Weiterhin war es nicht möglich, die nichttraumatisierte Kontrollgruppe des ersten Untersuchungszeitpunkts erneut zu untersuchen. Daher sind die in dieser Studie berichten hohen Komorbiditäten nur begrenzt als Traumafolgen zu interpretieren. Insgesamt war jedoch die Wiederteilnahmerate von 85\% der 1994 untersuchten Personen ein sehr erfreulicher Befund, der dafür spricht, dass die Studienteilnehmer selbst ein hohes Interesse an der Aufklärung der untersuchten psychologischen Zusammenhänge hatten, 
und daran, dass die Geschichte der politischen Inhaftierungen in der DDR nicht vergessen wird.

\section{Fazit für die Praxis}

Für die Begutachtung psychischer Folgen potentiell traumatisierender Ereignisse sowie für die klinische Praxis lässt sich schlussfolgern:

- Der Verlauf psychischer Folgen von potentiell traumatisierenden Ereignissen ist sehr variabel und in der Praxis sind deutlich mehr Verlaufsformen zu beobachten, als in den Klassifikationssystemen ICD-10 und DSM-IV aufgeführt.

- Selbsteinschätzungen der zu untersuchenden Personen sind nur unter Vorbehalt zu verwerten (vgl. Bodenburg \& Fabra, 2011) und regelhaft durch Sekundärdaten zu ergänzen. In retrospektiven subjektiven Beurteilungen können möglicherweise Aggravationstendenzen auftreten, die jedoch in subjektiven Beurteilungen der aktuellen Befindlichkeit weniger wahrscheinlich sind.

- Nach potentiell traumatisierenden Ereignissen leidet ein signifikanter Anteil der betroffenen Personen unter einer PTBS. Es findet sich aber auch ein hoher Anteil depressiver Störungen und verschiedener Angststörungen, die komorbid oder aber singulär auftreten. Dieser Befund deutet erneut darauf hin, dass der Fokus, sowohl im klinischen als auch im gutachtlichen Bereich, nicht auf das Auftreten posttraumatischer Belastungsreaktionen begrenzt werden kann (vgl. Foerster \& Widder, 2011). 


\section{Danksagung}

Die Autoren danken Maximilian Geiger, BSc, für seine Mitarbeit bei einem Teil der statistischen Analysen. 


\section{Literatur}

Bodenburg, S., Fabra M. (2011). Psychologische Selbsteinschätzungsskalen in gutachtlichen Untersuchungen. Nervenarzt 82: 910-916.

Brewin, C. R., Andrews, B., \& Valentine, J. D. (2000). Meta-Analysis of Risk Factors for Posttraumatic Stress Disorder in Trauma-Exposed Adults. Journal of Consulting and Clinical Psychology, 68(5), 748-766.

Dirkzwager AJ, Bramsen I, van der Ploeg HM. (2001). The longitudinal course of posttraumatic stress disorder symptoms among aging military veterans. Journal of Nervous Mental Disease, 189, 846-853.

Ehlers, A., Maercker, A. \& Boos, A. (2000) Predictors of chronic PTSD following political imprisonment: The role of mental defeat, alienation, and perceived permanent change. Journal of Abnormal Psychology, 109, 45-55.

Elhai JD, Palmieri PA. (2011). The factor structure of posttraumatic stress disorder. Journal of Anxiety Disorders, 25, 849-854.

Freyberger, H.J., Frommer, J., Maercker, A. \& Steil, R. (2003). Gesundheitliche Folgen politischer Haft in der DDR - Expertengutachten. Dresden: Konferenz der Landesbeauftragten für die Unterlagen des Staatssicherheitsdienstes der ehemaligen DDR.

Flatten, G., Gast, U., Hofmann, A., Knaevelsrud, C., Lampe, A., Liebermann, P., Maercker, A., Reddemann, L. \& Wöller, W. (2011). S3-Leitlinie Posttraumatische Belastungsstörung (ICD-10: F43.1). Trauma \& Gewalt, 5, 202-210.

Foerster K., Widder B. (2011). Begutachtung psychischer Unfallfolgen. Nervenarzt, $82: 15576-1565$.

Gäbler, I. \& Maercker, A. (2011). Revenge phenomena and PTSD in former East German political prisoners. Journal of Nervous and Mental Disease, 199, 287-294. 
Glück, T. \& Maercker, A. (2012). PTSD in the Elderly: An update on prevalence, symptom presentation, and clinical implications. Traumatic Stress Points, 26, 11-13.

Kessler, R. C., Sonnega, A., Bromet, E., Hughes, M., \& Nelson, C. B. (1995). Posttraumatic stress disorder in the National Comorbidity Survey. Archives of General Psychiatry, 52(12), 1048-1060.

Kleim, B. , Griffith, J.W., Gaebler, I., Schützwohl, M. \& Maercker, A. (2012). Political imprisonment shapes autobiographicak memory. Manuscript submitted for publication.

Krammer, S., Fankhauser, S., Günter, C. Schützwohl, M. Gäbler, I. \& Maercker, A. (2012). Social loss spiral: PTSD symptoms in former political prisoners and the role of socialinterpersonal factors. Manuscript submitted for publication.

Lapp, L.K., Agbokou, C. \& Ferreri, F. (2011). PTSD in the elderly: the interaction between trauma and aging. International Psychogeriatrics, 23, 858-868.

Maercker, A. (1999). Lifespan psychological aspects of trauma and PTSD: Symptoms and psychosocial impairments. In A. Maercker, M. Schützwohl \& Z. Solomon (Eds.), Posttraumatic stress disorder. A lifespan developmental perspective (pp. 7-42). Seattle: Hogrefe \& Huber.

Maercker, A., Beauducel, A., \& Schützwohl, M. (2000). Trauma severity and initial reactions as precipitating factors for posttraumatic stress symptoms and chronic dissociation in former political prisoners. Journal of Traumatic Stress, 13, 651-660.

Maercker, A., Gäbler, I., O'Neil, J., Schützwohl, M. \& Müller, M. (2012). Long-term trajectories of PTSD or resilience in former East German political prisoners. Manuscript submitted for publication.

Maercker, A., \& Horn, A. B. (2012). A socio-interpersonal context model of PTSD: the case for environments and interpersonal processes. Clinical Psychology \& Psychotherapy, doi: 10.1002/cpp.1805. 
Maercker, A., \& Schützwohl, M. (1997). Psychological long-term effects of political imprisonment: A group comparison study. Social Psychiatry and Psychiatric Epidemiology, 32, 434-442.

Morina N, Böhme HF, Morina L, Asmundson GJ. (2010). The structure of post-traumatic stress symptoms in young survivors of war. Psychiatric Research, 186, 306-309.

Morina, N., Böhme, H.F., Adjukovic, D., Bogic, M., Franciskovic, T. Galeazzi, G.M., Kucukalic, A., Lecic-Tosevski, D., Popovski, M, Schützwohl, M., Stangier, U. \& Priebe, S. (2010). The structure of post-traumatic stress symptoms in survivors of war: Confirmatory factor analyses of the Impact of Event Sclae - Revised. Journal of Anxiety Disorders 24: 606-611.

Ost LG, Alm T, Brandberg M, Breitholtz E. (2001). One vs five sessions of exposure and five sessions of cognitive therapy in the treatment of claustrophobia. Behaviour Research and Therapy, 39, 167-183.

Perkonigg, A., Pfister, H., Stein, M. B., Höfler, M., Lieb, R., Maercker, A. \& Wittchen, H. U. (2005). Longitudinal course of PTSD and PTSD symptoms in a community sample of adolescents and young adults. American Journal of Psychiatry, 162, 1320-1327.

Priebe, S., Rudolf, H., Bauer, M. \& Häring, B. (1992). Psychische Störungen nach politischer Inhaftierung in der DDR - Sichweisen der Betroffenen. Fortschritte der Neurologie und Psychiatrie, 61, 55-61.

Prot, K. (2009). Late effects of trauma: PTSD in Holocaust survivors. Journal of Loss and Trauma, 15, 28-36.

Schäfer I (2011) Traumatisierung und Sucht. In: Seidler G, Freyberger H, Maercker A, (Hrsg.) Handbuch Psychotraumatologie. Stuttgart: Klett-Cotta. S. 255-263.

Schneider, S., \& Margraf, J. (2006). DIPS-Diagnostisches Interview bei psychischen Störungen [Diagnostic interview for mental disorders] (3 ed.). Göttingen: Hogrefe. 
Schützwohl, M., \& Maercker, A. (1999). Effects of varying diagnostic criteria for posttraumatic stress disorder are endorsing the concept if partial PTSD. Journal of Traumatic Stress, 12, 155-165.

Solomon, Z. (1994). Coping with war-induced stress. New York: Plenum.

Solomon, Z., \& Mikulincer, M. (2006). Trajectories of PTSD: A 20-Year Longitudinal Study. American Journal of Psychiatry, 163(4), 659-666.

Solomon, Z., \& Mikulincer, M. (2007). Posttraumatic intrusion, avoidance, and social functioning: A 20-year longitudinal study. Journal of Consulting and Clinical Psychology, $75(2), 316-324$.

Uchino BN, Birmingham W, Berg CA. (2010). Are older adults less or more physiologically reactive? A meta-analysis of age-related differences in cardiocvascular reactivity to laboratory tasks. Journals of Gerontology B Psychol Sci Soc Sciience, 65B, 154-162.

Weissflog, G., Klinitzke, G. \& Hinz, A. (2011). Gesundheitsbezogene Lebensqualität und posttraumatische Belastungsstörungen bei in der DDR politisch Inhaftierten. Psychotherapie, Psychosomatik, Medizinische Psychologie, 61, 133-139.

Yehuda, R., Schmeidler, J., Labinsky, E., Bell, A., Morris, A., Zemelman, S., et al. (2009). Ten-year follow-up study of PTSD diagnosis, symptom severity and psychosocial indices in aging holocaust survivors. Acta Psychiatrica Scandinavica, 119, 25-34. 
Tabelle 1.

Soziodemographische Merkmale der ehem. politischen Inhaftierten und Drop-out-Analyse (vom Zeitpunkt der Ersterhebung)

\begin{tabular}{|l|l|l|l|}
\hline & $\begin{array}{l}\text { Teilnehmer beider } \\
\text { Erhebungszeitpunkte } \\
\mathrm{N}=93\end{array}$ & $\begin{array}{l}\text { Drop-outs } \\
\mathrm{N}=53\end{array}$ & $\begin{array}{l}\text { Testung } \\
\mathrm{t}-/ \chi 2-\text { Test (p- } \\
\text { Werte) }\end{array}$ \\
\hline Alter & $51.26 \pm$ 10.59 [27-72] & $57.74+12.95[28-82]$ & $3.24(0.001)^{*}$ \\
\hline Geschlecht (\% männl.) & 83.9 & 88.7 & $0.63(0.426)$ \\
\hline Bildungsabschlüsse (\%) & & & $5.699(0.017)^{*}$ \\
Hauptschule/mittl. Reife & 52.7 & 73.5 & \\
Abitur/Studium & 47.3 & 26.5 & $16.4(0.000)^{* *}$ \\
\hline Erwerbstätigkeit (\%) & & & \\
In Arbeit & 48.3 & 18.4 & \\
Arbeitslos & 23.6 & 20.4 & $1.25(7.42)$ \\
Berentet & 28.1 & 61.2 & \\
\hline Partnerschaftsstatus \% & & & \\
Alleinstehend & 13.2 & 14.3 & \\
Verheiratet/Zusammenl. & 60.4 & 55.1 & \\
Geschieden & 22.0 & 28.6 & \\
Verwitwet & 4.4 & 2.0 & \\
\hline
\end{tabular}


Tabelle 2. Prävalenzen psychischer Störungen im längsschnittlichen Vergleich

\begin{tabular}{|c|c|c|c|c|c|}
\hline & \multicolumn{2}{|l|}{1994} & \multicolumn{2}{|l|}{2008} & \multirow{2}{*}{$\begin{array}{l}\text { McNemar } \chi 2: \\
p \text {-Wert }\left(N_{\text {valid }}\right)\end{array}$} \\
\hline & $\mathrm{N}$ & $\%$ & $\mathrm{~N}$ & $\%$ & \\
\hline PTBS, aktuell & 25 & 29.1 & 28 & 32.6 & $0.7(86)$ \\
\hline $\begin{array}{l}\text { Agoraphobie ohne } \\
\text { Panikstörung }\end{array}$ & 10 & 11.6 & 8 & 9.3 & $0.8(86)$ \\
\hline $\begin{array}{l}\text { Panikstörung } \\
\text { mit/ohne } \\
\text { Agoraphobie }\end{array}$ & 22 & 25.8 & 21 & 24.4 & $0.9(86)$ \\
\hline $\begin{array}{l}\text { Spezifische Phobie, } \\
\text { Klaustrophobischer } \\
\text { Typ }\end{array}$ & 18 & 21.2 & 2 & 2.4 & $0.000(85)^{* *}$ \\
\hline $\begin{array}{l}\text { Andere spezifische } \\
\text { Phobien }\end{array}$ & 14 & 16.3 & 6 & 7 & $0.1(86)$ \\
\hline Sozialphobie & 11 & 12.8 & 6 & 7 & $0.13(86)$ \\
\hline $\begin{array}{l}\text { Generalisierte } \\
\text { Angststörung }\end{array}$ & 5 & 5.8 & 5 & 5.8 & $1.0(86)$ \\
\hline OCD & 2 & 2.3 & 3 & 3.5 & $1.0(86)$ \\
\hline $\begin{array}{l}\text { Major Depression, } \\
\text { aktuelle Episode }\end{array}$ & 5 & 5.8 & 23 & 25.6 & $0.000(86)^{*}$ \\
\hline $\begin{array}{l}\text { Major Depression, } \\
\text { rezidivierend }\end{array}$ & 10 & 11.6 & 15 & 16.3 & $0.31(86)$ \\
\hline Dysthymie & 5 & 5.8 & 5 & 5.8 & $1.0(86)$ \\
\hline $\begin{array}{l}\text { Somatoforme } \\
\text { Störungen }\end{array}$ & 14 & 18.9 & 14 & 18.9 & $1.0(74)$ \\
\hline $\begin{array}{l}\text { Substanzmissbrauch/ } \\
\text {-abhängkeit }\end{array}$ & 13 & 14.0 & 3 & 3.5 & $0.007(86)^{*}$ \\
\hline
\end{tabular}


Tabelle 3. Vergleich von Traumafolgen-Verlaufstypen über drei Zeitpunkte (T0, T1, T2; Erläuterungen im Text) zwischen Klinker-Beurteilung und Teilnehmer-Selbsteinschätzung

\begin{tabular}{|c|c|c|c|}
\hline Verlaufsmuster & $\begin{array}{l}\text { Kliniker-Beurteilung } \\
\text { (retro- und } \\
\text { prospektiv) }\end{array}$ & $\begin{array}{l}\text { Teilnehmer- } \\
\text { Selbsteinschätzung } \\
\text { (retrospektiv) }\end{array}$ & $\begin{array}{l}\text { Übereinstimmung } \\
\text { bezogen auf } \\
\text { Klinker-Beurteilung }\end{array}$ \\
\hline & $\mathrm{N}(\%)^{1}$ & $\mathrm{~N}(\%)^{1}$ & $N(\%)^{2}$ \\
\hline Chronisch & $14(16.3)$ & $16(18.3)$ & $6(42.9)$ \\
\hline Resilient & $26(30.2)$ & $31(36.0)$ & $16(61.5)$ \\
\hline verzögert & $6(7.0)$ & $13(15.2)$ & \\
\hline früh verzögert & $1(1.2)$ & $12(14.0)$ & -3 \\
\hline spät verzögert & $5(5.8)$ & $1(1.2)$ & $0(0)$ \\
\hline remittiert & $32(37.2)$ & $19(22.1)^{*}$ & \\
\hline früh remittiert & $22(25.6)$ & $11(12.8)$ & $5(22.7)$ \\
\hline spät remittiert & $10(11.6)$ & $8(9.3)$ & $2(20.0)$ \\
\hline wechselnd & $8(9.3)$ & $7(8.1)$ & \\
\hline umgekehrt U-förmig & $0(0)$ & $5(5.8)$ & -3 \\
\hline U-förmig & $8(9.3)$ & $2(2.3)$ & $0(0)$ \\
\hline
\end{tabular}

Anmerkung: ${ }^{1}$ bezogen jeweils auf $\mathrm{N}=86 ;{ }^{2}$ Prozentangabe bezogen jeweils auf Kliniker-Beurteilung (1. Spalte); ${ }^{3}$ zu geringes $\mathrm{N}$ bei der Kliniker-Beurteilung; ${ }^{*} \mathrm{p}<0.05$ für Vergleich Kliniker-

Teilnehmerbeurteilung 
Abbildung 1: Vergleich der PTBS-Symptomprofile zu T1 und T2

Symptomprofile PTBS T1 und T2

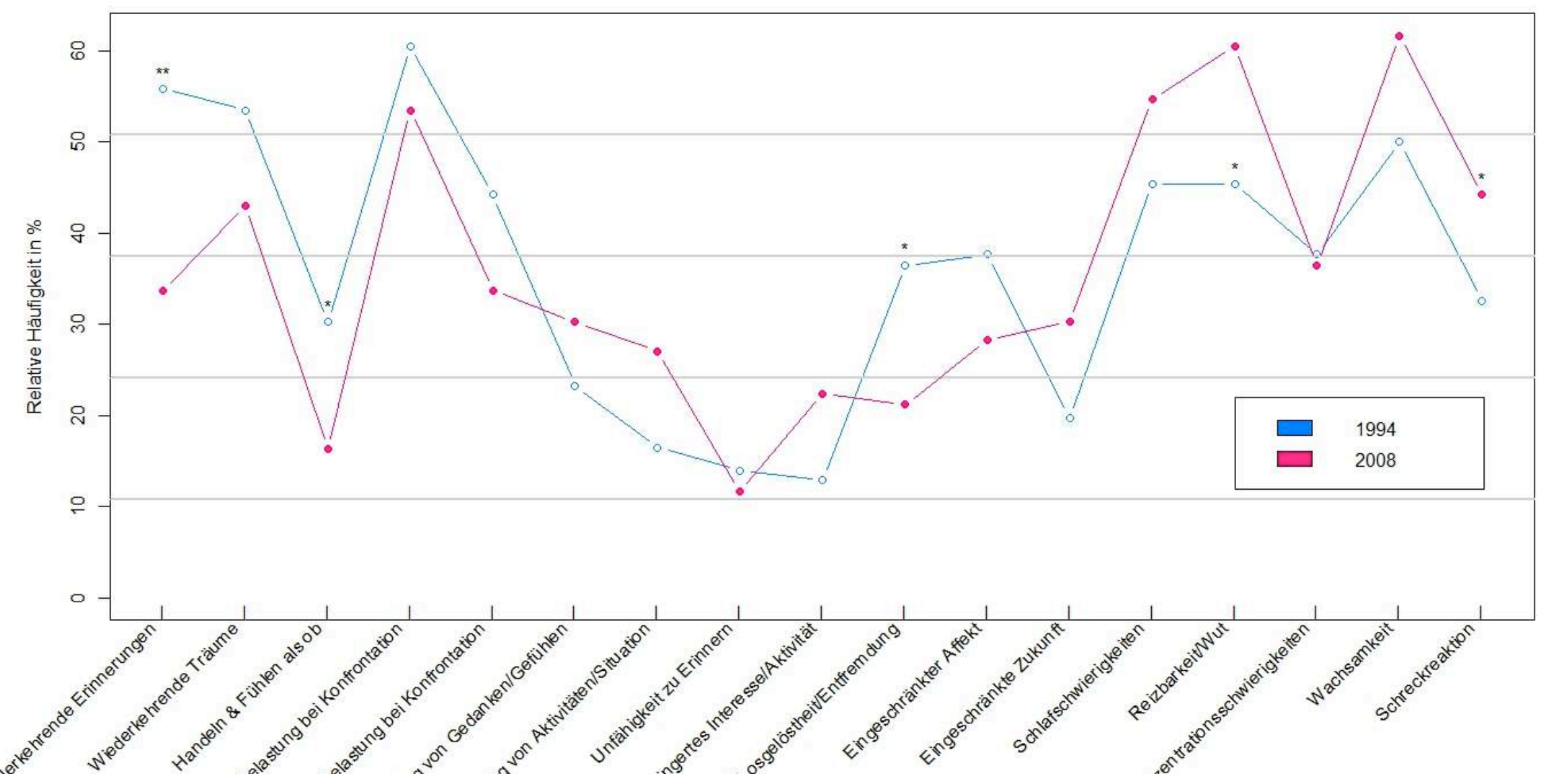

